\title{
Darwin, Éros et moi, ou comment la psychologie évolutionniste m'a incité à lire et invité à écrire les histoires d'amour
}

\author{
Martin Winckler \\ Écrivain résidant à Montréal (P.O.L)
}

Comme beaucoup de Français, j'ai longtemps baigné dans une vision du monde sur-psychanalysée et, de ce fait, paralysante. Chacun de mes gestes, chacune de mes phrases, chacune de mes pensées, à peine exprimées, faisaient l'objet d'une interprétation, le plus souvent sauvage, de la part de tout un chacun - enseignants, camarades, collègues bien- ou malveillants - et même parfois de l'un de mes proches. Pour avoir vécu la montée du féminisme dans les années 1970, j’ai pu constater à quel point les relations entre hommes et femmes pouvaient, au prisme d'une théorie freudienne parfois 
incompréhensible - et qu'il était, le plus souvent, impossible de mettre en question - devenir suspectes. J'en avais conclu un peu hâtivement, mais qui étais-je pour contester Freud et Lacan? -, que tout homme cache un violeur en sommeil, que toute femme aimant la sexualité est probablement hystérique et que tout enfant rêve de coucher avec un de ses parents. Ma réflexion personnelle sur les relations d'amour s'en trouvait singulièrement compliquée, aussi bien dans ma profession de médecin (quelle consultation ne renvoie pas à la sexualité, de près ou de loin ?) que dans mon travail d'écrivain: depuis l'adolescence, mon rêve d'écrire un roman d'amour ressemblait à une mission impossible.

$\mathrm{Au}$ début des années 2000, à l'occasion de voyages fréquents en Amérique du Nord, j'ai eu accès à un pan de savoir et d'exploration intellectuelle qui m'était jusque-là inconnu : la psychologie évolutionniste. Au travers d'ouvrages tels que The Anatomy of Love de Helen Fisher ou The Mating Mind de Geoffrey Miller, je découvrais que, pour les anthropologues et psychologues évolutionnistes, les comportements amoureux ne sont pas "pervers » ou " anormaux » mais obéissent à des lois régissant l'ensemble du règne animal. En effet, les deux impératifs de l'évolution - survivre et se reproduire - ont contribué à sélectionner des mécanismes communs à toutes les espèces sexuées, y compris homo sapiens. Dans notre espèce comme dans bien d'autres, ces mécanismes diffèrent entre mâles et femelles, parce que le coût de la reproduction n'est pas le même pour les membres des deux sexes. Pour assurer la survie de leurs gènes et leur passage dans les générations à venir, les hommes oscillent entre deux stratégies: soit augmenter le nombre de leurs descendants en séduisant un grand nombre de partenaires, soit maximiser les chances de 
survie d'un nombre plus petit en privilégiant une relation monogame. Pour assurer la survie de leur progéniture, les femmes, quant à elles, oscillent entre deux stratégies très différentes mais souvent complémentaires: rechercher des gènes de très bonne qualité auprès des mâles très convoités et souvent volages - ou opter pour un partenaire de moindre qualité génétique, mais qui se révélera un soutien solide, un pourvoyeur efficace et un protecteur sûr.

De plus, l'humanité a hérité d'un privilège (ou d'un malheur) insigne: en dehors des dauphins et des singes bonobos, les êtres humains semblent être les seuls pour qui les contacts sexuels sont agréables et désirables même en dehors de toute pulsion reproductive. C'est la principale raison qui les fait recommencer. Les mantes religieuses mâles, sacrifiés par la femelle au moment du coït, n'ont pas le même loisir.

\section{Sexe, séduction et sociétés}

Chez les humains, les choix des membres de l'un et de l'autre sexe sont de plus modelés par la structure sociale : ainsi, une société fortement hiérarchisée est associée à la polygamie des hommes les plus puissants et au célibat des hommes les plus pauvres, tandis qu'une société plus égalitaire penche plutôt vers la monogamie. Cette vision des choses, de nombreux chercheurs, anthropologues, ethnologues, sociologues, l'ont confirmée dans toutes les cultures étudiées (voir Buss). On en a déduit que les comportements sexuels ne sont pas en euxmêmes culturels, mais innés. C'est leur mise en scène, ellemême la résultante de l'environnement propre à chaque groupe 
humain, qui est le produit de la "culture », laquelle interagit à son tour sur la sexualité au fil des transformations sociales.

Ainsi, comme le montre Sarah Blaffer Hrdy dans son ouvrage fondamental, Mother Nature, le viol, l'infanticide, l'abandon d'enfant et l'avortement sont des comportements transculturels qui ont toujours existé et correspondent à des stratégies de reproduction inconscientes, observables dans des espèces aussi diverses que la souris, le lion ou les singes capucins.

De même, les stratégies de séduction varient selon le sexe, ce que Darwin suggérait déjà au travers des différences entre des paons au plumage imposant et énergétiquement coûteux, et des femelles physiquement beaucoup moins attrayantes. Dans son ouvrage Spent. Sex, Evolution and Consumer Behavior, Geoffrey Miller, encore lui, explique ainsi avec beaucoup d'humour que la production multimillénaire d'œuvres artistiques - de la pierre taillée aux concerts de rock en passant par la sculpture, la composition musicale et le roman - et l'affichage outrancier de signes extérieurs de santé et de richesse sont au fond équivalents aux queues des paons pour les hommes désireux d'attirer l'attention d'éventuelles partenaires sur la qualité de leur génome. Ces manifestations de fitness display et de conspicuous consumption sont très probablement propres à chaque genre. Les structures sociales qui restreignent aujourd'hui les possibilités d'expression des femmes seraient la conséquence des modifications sociales entraînées par le développement de l'agriculture, il y a huit mille ans. 


\section{Freud au rencart}

Face aux travaux nombreux et convaincants publiés par les psychologues évolutionnistes au cours des trente dernières années, les «lois » freudiennes - à commencer par leur tarte à la crème, le complexe d'CEdipe - se voient peu à peu disqualifiées en tant qu'explication des modes de pensée inconscients (voir Scalise Sugiyama, p. 306-315). Elles semblent d'ailleurs avoir complètement disparu de la pensée dans les pays développés et survivre exclusivement - et assez inexplicablement, remarque Dylan Evans dans son article «From Lacan to Darwin » (dans Gottschall et Wilson, p. 38-55) - en France et en Argentine.

À mesure que je m'éloignais de la théorie freudienne et progressais dans ma compréhension des mécanismes psychologiques décrits - preuves statistiques à l'appui - par les chercheurs évolutionnistes, je m'intéressais également au regard porté par d'autres chercheurs darwiniens sur la littérature et les arts. Parmi les ouvrages les plus récents, j'ai été très frappé par The Art Instinct, de Denis Dutton, et par On the Origin of Stories, de Brian Boyd. Ce dernier avance, avec des arguments très convaincants, que la narration, capacité exclusivement humaine de composer et d'écouter des récits, serait, comme la bipédie et le langage, un produit de l'évolution, engrammé dans nos fonctions cérébrales innées. La narration permet en effet non seulement de transmettre des informations pratiques, des valeurs et des sentiments, mais aussi d'exposer l'auditeur (ou, aujourd'hui, le spectateur et le lecteur) à des situations de vie qu'il n'a pas pu vivre encore et, ce faisant, de le préparer à faire face à celles qu'il rencontrera. Autrement dit, 
de même que les jeux de lutte ou de course auxquels s'adonnent les animaux est un entraînement à la chasse, au combat ou à la fuite, les histoires constitueraient, pour le cerveau des humains, un entraînement à la vie. Dans cette perspective, toute œuvre littéraire aurait pour but implicite et inconscient de décrire des comportements humains pouvant servir d'« exemples », positifs ou négatifs, au public qui les reçoit. Les analyses littéraires évolutionnistes sont aujourd'hui nombreuses. Evolution, Literature and Film, anthologie composée par Boyd, Carroll et Gottschall, trois spécialistes de cette jeune discipline, nous propose par exemple des études sur L'Odyssée, Othello, Great Expectations et Citizen Kane.

En tant que lecteur et écrivant, mon intérêt s'est toujours porté préférentiellement sur le récit, la narration, la construction des histoires plutôt que sur le style d'écriture ou les «complexes freudiens» d'un personnage. L'approche évolutionniste m'a incité à entreprendre, à mon tour, une lecture d'œuvres populaires connues. En voici deux exemples.

\section{"We'll always have Paris ": une lecture évolutionniste de Casablanca de Michael Curtiz (1942)}

Paris, printemps 1940. Persuadée que son mari Viktor Laszlo (Paul Henreid) est mort en déportation, Ilsa Lund (Ingrid Bergman) rencontre Nick Blaine (Humphrey Bogart) et en tombe amoureuse. Au bout de quelques semaines, leur bonheur est assombri par l'annonce de la capitulation. Le jour de l'entrée des Allemands à Paris, Ilsa disparaît sans explication. Désespéré, Rick prend le dernier train d'Austerlitz et quitte le 
territoire français. Deux ans plus tard, à Casablanca, il voit Ilsa entrer dans son établissement, le Rick's Café, au bras de Viktor, bien vivant. Le couple est en fuite. Chef de la résistance, Laszlo doit quitter le Maroc au plus vite pour échapper aux Allemands et poursuivre la lutte. Or, Rick est en possession de deux saufconduits. Un soir, après lui avoir proposé, sans succès, de les lui acheter, Ilsa s'offre à son ancien amant et lui promet de rester avec lui s'il donne à Viktor la possibilité de s'enfuir. Rick lui reproche de l'avoir trompé et refuse un marché indigne de leur amour passé. Elle le menace d'une arme, mais il lui déclare que, depuis qu'elle l'a quitté, sa vie n'a plus de sens. Elle défaille, car elle l'aime toujours, elle aussi. Ils tombent dans les bras l'un de l'autre. Certes, au petit matin, la caméra les retrouve debout dans la même pièce, la veste de Bogart n'a pas le moindre pli, la coiffure de Bergman pas une mèche de travers (la censure de l'époque avait imposé au réalisateur de laisser planer le doute) mais le spectateur présume qu'ils ont passé la nuit ensemble. Et il a parfaitement raison.

Ilsa Lund se trouve en effet confrontée à un dilemme darwinien typique. Rick l'aventurier incarne la force, l'énergie sexuelle et la fertilité. Laszlo est la figure tutélaire synonyme d'intelligence et de rigueur morale mais, parce qu'il a été torturé dans un camp de concentration, on peut supposer que sa vitalité sexuelle et sa fertilité ont été altérées, de manière peut-être irréparable. En s'offrant à Rick en échange du saufconduit, Ilsa opère une stratégie reproductive qui, quoiqu'inconsciente, est tout à fait appropriée. Elle n'est pas sûre de pouvoir être enceinte de son mari. En se donnant à Rick malgré son refus de les aider, Ilsa saisit l'occasion de mêler ses gênes à ceux de son amant, ce qui compense largement la perspective de rester coincée à Casablanca avec Viktor. 
Lorsque le jour se lève sur les deux amants, ils semblent avoir renoué : après avoir donné un sauf-conduit à Laszlo, Rick et Ilsa quitteront Casablanca ensemble. D'ailleurs, on voit le héros s'empresser de vendre le Rick's Café à son principal concurrent afin d'amasser de l'argent le plus vite possible. Mais le soir suivant, coup de théâtre! Lorsque le trio arrive à l'aéroport, Rick révèle ses intentions véritables. Il a depuis le début décidé de donner les sauf-conduits au couple et de les laisser partir ensemble. Tandis que les moteurs du dernier avion se mettent à tousser, Rick explique à la jeune femme stupéfaite que les enjeux (la survie d'un chef de la résistance) dépassent leur petite personne. Viktor aura besoin d'elle. Tout en l'assurant de son amour («We'll always have Paris »), il la convainc de s'envoler avec son mari. Lorsque Strasser, l'officier allemand, tente de s'interposer, Rick le tue sans hésiter, au risque de se faire lui-même arrêter par la police française.

Si, comme le déclare solennellement Rick à Viktor avant de les pousser vers l'avion, Ilsa ne lui est pas tombée dans les bras, la conduite sacrificielle du personnage incarné par Bogart est moralement admirable... mais stérile. Si, en revanche, Rick a menti pour préserver la relation entre Ilsa et Viktor, son «sacrifice » revêt une tout autre dimension.

Car en sauvant Ilsa et son mari, Rick ne sauve pas seulement le monde libre, il sauve l'enfant possible d'Ilsa, un enfant dont les chances de survie sont bien meilleures avec le mari légitime en Amérique du sud qu'avec l'amant traqué en Afrique du Nord. Rick ne se sacrifie pas, il fait lui aussi, comme la femme qu'il aime, un choix judicieux. Car l'enfant que portera Ilsa sera peut-être le sien. 
Viktor, figure héroïque, n'est pas lésé pour autant. S'il n'est pas stérile, il aura d'autres enfants avec cette femme admirable. S'il est stérile, il a bénéficié d'une insémination par un donneur de grande qualité. L'enfant à naître sera fils de Viktor et de Rick. Sur le plan symbolique comme sur le plan génétique, tout le monde est gagnant.

\section{Cyrano, Christian, Roxane et l'évolution}

Un soir, en dégustant le magnifique Cyrano de Bergerac que Denis Podalydès mit en scène à la Comédie-Française en 20061, je me suis écrié : «Cyrano est darwinien!»

Comme la littérature l'a toujours proclamé, les femmes ne se laissent pas séduire facilement et, pour les conquérir, les hommes doivent ramer. La psychologie évolutionniste nous apprend, de plus, que les critères de choix respectifs ne sont pas les mêmes. Les hommes préfèrent les femmes jeunes et physiquement attirantes (signes de santé et de fertilité); les femmes préfèrent les hommes plus âgés qu'elles et déjà établis dans la vie (assurance de sécurité matérielle pour elles et leurs enfants). Mais elles veulent aussi des hommes beaux et intelligents, traits génétiques hautement désirables, et qui plus est, loyaux et fiables. La barre est haute. Alors, de même que les paons font la roue, certains hommes affichent leur « patrimoine génétique » en faisant du sport pour montrer leurs muscles; d'autres encore en roulant dans de grosses voitures pour montrer qu'ils ont beaucoup d'argent; d'autres, enfin, en composant des pièces de théâtre ou en faisant des vers.

\footnotetext{
${ }^{1}$ Le DVD est disponible aux éditions Montparnasse.
} 
Et nous voici de retour à Rostand.

Roxane est adorée du beau mais sot Christian.

Elle est aussi aimée - en secret, cependant, par Cyrano, héros aussi laid que brillant.

Roxane tient en horreur, et bêtise, et laideur.

Brusquement, Cyrano tend un pacte à Christian :

"Lions mon éloquence à ton charme vainqueur,

et faisons à nous deux un héros de roman!»

Charmée par les mots doux que Cyrano lui trousse, Roxane cède, éperdue, à Christian et l'épouse.

Mais alors, direz-vous ! Cyrano est perdant !

Eh bien, pas tout à fait. Car voyez-vous, les lois de l'évolution veulent qu'un individu, à défaut de pouvoir se reproduire, favorise dans certaines situations la reproduction d'un individu apparenté (porteur de certains gènes identiques aux siens). Or, Roxane et Cyrano sont... cousins ! En favorisant l'union de la belle à Christian, Cyrano permet à une partie de ses gènes (celle qu'ils ont en commun) de passer à la postérité. Simple, et élégant!

Si le jaloux de Guiche, prétendant de naguère

N'expédiait l'époux se faire tuer à la guerre,

Roxane et Christian auraient beaucoup d'enfants.

\section{Un projet de roman darwinien}

Voir en ces classiques du cinéma américain et de la littérature française une illustration de l'altruisme inclusif suggéré par Darwin et développé plus récemment par Trivers est à mes yeux plus enthousiasmant (et bien moins nez-buleux) que l'explication freudienne selon laquelle Ilse choisirait de coucher avec son père ou qu'une analyse lacanienne du symbole phallique saillant sous le chapeau du héros de Rostand. 
Je caresse actuellement l'idée de deux projets. Le premier consisterait en une série de "lectures » similaires aux deux précédentes, consacrées à des films, à des séries télévisées et à des pièces de théâtre célèbres et rassemblées dans des livres intitulés «Darwin à l'écran » ou « Darwin en scène ». Le second projet est celui d'un roman. Dans l'esprit des propos tenus par Brian Boyd dans On the Origin of Stories, il me semble en effet qu'une fiction serait le meilleur moyen de présenter les principes de la psychologie évolutionniste au public français sous une forme à la fois ludique, signifiante et pédagogique.

Ce roman serait constitué de trois narrations parallèles : - un séminaire de psychologie évolutionniste mettant en face-àface un professeur chevronné et une quinzaine d'étudiants « innocents »;

- la fiction documentaire que l'enseignant projette au cours du séminaire pour illustrer les différentes stratégies de reproduction des hommes et des femmes ;

- le récit des répercussions de ce cours sur les étudiants, entre deux séances.

Le titre, Les Démons, désignerait non seulement certains personnages du roman (et du film-dans-le-roman) mais aussi les «génies», «esprits», «familiers» et autres êtres imaginaires et invisibles auxquels les humains demandent conseil et qui, parfois, les inspirent, car ils sont engrammés dans leurs cerveaux.

Ce roman est encore à l'état de projet et d'esquisses, mais en voici quelques fragments. 
LE PROGRAMME, 1

Département de psychologie évolutionniste

Session d'hiver

Septembre-décembre

Cours EVO 7007

«Stratégies de choix des partenaires sexuels »

Professeur : John L. Cosby

Jeudi, 16 h - 19 h

Présentation du cours

Séance 1,6 septembre :

- Introduction, discussion du plan de cours, description des devoirs, répartition des présentations (chaque étudiant présentera un concept de la psychologie évolutionniste en début de séance).

- Rappels sur la théorie de l'évolution. Notion de "gène égoïste ». Fondements de la psychologie évolutionniste.

Lectures préparatoires : Workman, Lance et Will Reader, Psychologie évolutionniste, trad. de l'anglais par Françoise Parot, Louvain-la-Neuve / Paris, de Boeck, 2007, section 1 (Introduction à la psychologie évolutionniste) et section 2 (Les mécanismes de l'évolution).

Séance 2, 13 septembre : Sélection sexuelle, 1

Lectures: Workman \& Reader, Psychologie évolutionniste, section 3.1 et 3.2

Présentation $1:$ «théorie de l'esprit » (Jessica B.)

LE SÉMINAIRE, 1

- Allez-y, Jessica, nous vous écoutons.

- Merci, Professeur. Alors, je commence... Excusez-moi, je vais lire ce que j'ai écrit, parce que je ne veux pas me tromper... Voilà : La théorie de l'esprit - theory of mind en anglais -, que Lapprand nomme "psychologie intuitive» dans son ouvrage sur Winckler, est l'un des concepts fondamentaux de la psychologie évolutionniste. C'est l'aptitude que les êtres humains ont de «lire dans les pensées » de leurs interlocuteurs 
- en tout cas de les deviner ou de les anticiper - en voyant leur visage, leur posture corporelle ou leur comportement, ou de deviner ce que leurs interlocuteurs pensent d'eux en les regardant. L'exemple le plus classique est celui de l'enfant qui vient de manger du chocolat à tartiner à même le pot, alors que sa mère le lui avait interdit. Quand celle-ci entre dans la cuisine, il a rangé le pot et caché la cuillère. Sa mère le regarde d'un air courroucé, mais ne dit rien. Il sait qu'elle est en colère et ne comprend pas tout de suite pourquoi. Mais il se doute bien de la raison, et quand il se passe la langue sur les lèvres, il se rend compte qu'il a encore du chocolat partout. Il sait — ou du moins postule avec une bonne chance d'avoir raison - que sa mère sait... (Rires.) L'aptitude à émettre ces hypothèses et ces déductions apparaît chez l'enfant entre l'âge de trois et cinq ans. La théorie de l'esprit a été testée et démontrée par de nombreuses équipes. La notion de son existence permet de décrire et d'analyser de communications non-verbales et aussi de comprendre les mécanismes par lesquels une personne est en mesure d'en manipuler une autre. [...] »

\section{LE FILM, 1}

Vous voyez l'homme assis au bar? Il a l'air plutôt nerveux, vous ne trouvez pas? Eh bien, il y a de quoi! Dans moins d'une heure, sa vie va basculer et il s'il le sentait confusément depuis plusieurs semaines, ce soir il en est convaincu. S'il est assis là ici dans un lieu qu'il n'a jamais fréquenté auparavant, c'est pour un motif qui, à défaut de pouvoir être qualifié de "légitime", est terriblement puissant.

Il passe son temps à regarder autour de lui, comme s'il craignait d'être reconnu. Pourtant, la probabilité est mince, et les conséquences éventuelles, insignifiantes. Le bar dans lequel il est assis n'a rien de louche; c'est celui du Royal Club, hôtel plutôt chic de Toronto où il passe la nuit pour la première fois. Il connaît peu de monde en ville, et personne qui séjourne à l'hôtel. Et, précisément, son malaise vient de là.

Il se nomme John Adamson. Il a cinquante ans, est professeur de gynécologie. Il est marié à Jane Evers, quarante-huit ans, professeure de droit dans la même université que son mari : McGill University, à Montréal. Le couple vit depuis vingt ans 
dans Notre-Dame-de-Grâce, un quartier anglophone aisé dans l'ouest de l'île avec leurs trois enfants : une fille aînée, Jill, et deux garçons, Dean et Sam. Les deux aînés sont étudiants, Sam encore à l'école secondaire.

Le malaise présent de John n'est pas, en soi, dû à son éloignement de Montréal et de sa famille. Ce n'est pas la première fois qu'il séjourne à Toronto. Depuis le début de l'année universitaire, il vient chaque mois y donner un séminaire de deux jours consacré à la santé des femmes. Il s'envole de Montréal le mardi matin, passe la nuit du mardi au mercredi dans la métropole ontarienne et repart au Québec le mercredi en fin d'après-midi. Pendant ces deux jours, au cours de quatre séances de trois heures, il s'adresse à une douzaine de professionnels de santé d'âges divers qui apprécient beaucoup son enseignement, son humour, ses qualités humaines et son sens pédagogique. Il entretient avec les participants du séminaire - médecins, cadres infirmiers, étudiants - une relation très gratifiante et il est probable que, lors des évaluations de fin de séminaire, tous lui attribueront une note excellente.

John repose son verre et tripote le téléphone cellulaire posé sur le comptoir. Il est dix-neuf heures. Il vient d'appeler Jane à Montréal, ce qui est inhabituel : il ne l'appelle jamais lorsqu'il passe la nuit à Toronto. Les mardis soirs de séminaire, il a l'habitude d'aller dîner avec ses étudiants dans un restaurant de Chinatown, non loin de l'Université, puis rentre dormir chez un couple d'amis enseignants de médecine, qui logent dans un appartement sur le campus. Mais aujourd'hui, nous sommes mercredi. C'est la deuxième nuit d'affilée que John passe au bord du lac Ontario. Il a donné aujourd'hui les dernières séances de séminaire de la saison et invoqué un repas d'adieux en expliquant à Jane, il y a déjà trois semaines, cette nuit d'absence supplémentaire.

- Le soir de la dernière rencontre, mes étudiants m'invitent à dîner.

- C'est adorable de leur part!

- Trois d'entre eux n'étaient pas libres le mardi soir ; alors, ils m'ont demandé si j'accepterais de rester une nuit de plus. 
John, qui se déplace régulièrement dans tout l'Est du pays pour donner cours et conférences, prévient toujours Jane de ses allées et venues longtemps à l'avance.

Mais lorsqu'il ne s'absente qu'une nuit, il n'appelle pas son épouse. Tout au plus lui envoie-t-il, au moment d'aller se coucher, un courriel qu'elle trouve au matin, en se réveillant.

Ce soir, cependant, il a éprouvé le désir de l'appeler. La conversation a été brève.

- Hey, Honey...

- Hey Darling! Are you all right?

Jane lui a semblé légèrement tendue, ce qu'il a attribué à la maladie de Brian, un de leurs amis les plus proches malade depuis plusieurs mois et qui ne quitte plus son domicile de Sherbrooke.

- Oui, oui, tout va bien, a déclaré John avec beaucoup d'assurance. Moi aussi, j'appelais pour savoir si tout va bien !

- Si quelque chose allait mal, il y aurait déjà dix messages sur ta boîte vocale! Tu avais peur que Brian...

- Non, non... J'appelais juste pour savoir si tout va bien à la maison.

- Oooh, comme c'est gentil... Comment se déroule ton dîner ? Où tes étudiants t'ont-ils emmené ?

- Je les attends. Je suis encore à l'hôtel.

- À l'hôtel ? Tu ne dors pas chez Nick et Nora?

- Non, les parents de Nora arrivaient aujourd'hui pour passer la fin de semaine avec eux. Ils m'ont proposé de dormir sur leur canapé, mais je n'ai pas voulu les encombrer.

- Tu as bien fait! Quoi ? Jill m'appelle! Nous allons au cinéma. Gotta go, Honey! Enjoy your dinner. Love you!

Après qu'elle a raccroché, John s'est senti à la fois soulagé et détestable. Car, pour la première fois en vingt ans de vie commune, il lui a menti. Ce qu'il a dit au sujet de Nick et Nora est vrai. Mais ce n'est pas pour cette raison qu'il a loué une chambre d'hôtel. Le repas d'adieux avec les étudiants a eu lieu hier soir.

John finit d'un trait son verre et fait signe au barman de lui resservir la même chose.

- Puis-je me joindre à vous ? demande une voix féminine. 


\section{LA DISCUSSION, 1}

Le professeur: Bon, on va s'arrêter là pour l'instant. Voulezvous rallumer les lumières, s'il vous plaît?

(Murmures déçus. Toussotements. Bruits de chaise.)

Le professeur: Bien! Avant d'analyser cette première séquence, des réactions? Ou des questions?

(Silence.)

Un étudiant : Ce sont des acteurs?

Le professeur: Bien entendu! Le film a été réalisé spécifiquement pour ce cours, à partir d'un scénario très précis. Une étudiante : Est-ce une histoire vécue?

Le professeur: Oh, je suis certain que des centaines de milliers de personnes ont vécu des situations similaires ! Cependant, les personnages sont imaginaires... D'autres questions ? Bien, alors vous avez lu les chapitres et les articles que je vous ai envoyés pour ce premier cours, j'imagine?

(Murmures embarrassés.)

Le professeur : (Soupir.) Bon... Qui peut me décrire la situation sociale de John ? (Silence.) Allez-y, lancez-vous, c'est en se trompant qu'on apprend la vérité. Oui ?... Josy, c'est ça ?

Josy : C'est ça.

Le professeur : Je vous écoute...

Josy: John est un homme installé, respectable, puisqu'il est médecin. Son statut social est important. Il vit avec une compagne dont le statut est élevé, également. Ils ont plusieurs enfants, on peut supposer qu'ils sont tous deux fertiles, mais à la fin de la période de reproduction, puisque, pour ce qui est tant de sa survie que de sa descendance, on peut considérer qu'il a réussi. Il semble entretenir une relation monogame avec sa compagne. Enfin, jusqu'ici.

Le professeur : «Jusqu'ici ». Que voulez-vous dire?

Josy : Sa compagne sait toujours où il se trouve, et il est très mal à l'aise de lui avoir menti pour la première fois. On peut penser qu'il est en train de cacher une relation extraconjugale. Mais s'il en avait déjà eu, il aurait été amené à lui mentir auparavant et ça ne lui serait pas aussi difficile. Par conséquent, c'est la première fois.

Le professeur : Pensez-vous que c'est obligatoire? 
Josy: De mentir pour avoir des relations extraconjugales? (Rire.) À moins de les annoncer à son épouse, oui...

Le professeur : Non, de mentir, tout simplement. Pensez-vous que c'est obligatoire?

Josy: Quand on veut tromper sa femme, c'est inévitable! (Rires.)

Le professeur : Inévitable de mentir?

Josy : En tout cas, c'est moralement inacceptable !

Le professeur: Souvenez-vous, nous ne sommes pas dans un cours de morale, ici, mais dans un cours de psychologie ! - Oui, Darryl ?

Darryl : Le mensonge fait partie des options comportementales sélectionnées par l'évolution. La possibilité de cacher, d'induire en erreur, est commune à tout le règne animal, aussi bien pour capturer une proie que pour échapper à un prédateur. Par conséquent...

Josy (énervée) : Mais John n'est pas menacé par un prédateur, là, il s'agit de sa femme !

Le professeur: C'est exact. Pourquoi donc a-t-il décidé de mentir?

Le groupe : Pour pouvoir la tromper !

Le professeur: Nous n'en savons rien! Pour le moment, c'est une supposition! Et son mensonge a une fonction plus immédiate, qui est... Ashley?

Ashley: De lui cacher qu'il reste à Toronto pour une autre raison. Afin d'éviter des représailles.

Le professeur : Des représailles?

Une voix non identifiée : Un divorce qui le laissera sur la paille! (Rires.)

Le professeur: Effectivement, c'est une possibilité. Mais on peut penser qu'en cet instant, ce n'est pas exactement cela qu'il redoute. Pourquoi ment-il, plus simplement ? Plus prosaïquement?

(Silence.)

Darryl : Parce qu'il ne veut pas que sa femme sache ce qu'il va faire de sa soirée.

Le professeur : Bien. Pourquoi?

Darryl : Parce qu'elle pourrait ne pas aimer ce qu'il va faire.

Le professeur: D'accord, mais ce qu'il va faire est-il inévitablement répréhensible? 
(Silence.)

Le professeur : Je reformule ma question. Ce mensonge signifiet-il nécessairement qu'il va tromper sa femme?

Josy : Non, mais il se comporte de manière suspecte.

Le professeur: Suspecte?

Josy : En appelant sa femme. Il ne le fait pas d'habitude.

Le professeur : Et cela signifie?

Darryl: Qu'il cherche à savoir si elle se doute de quelque chose... et qu'il se sent coupable.

Le professeur : Très bien. Qui pense que sa femme se doute de quelque chose ?... Qui pense le contraire ? Une majorité d'entre vous pensent que Jane se doute de quelque chose. Eh bien, nous allons tout de suite découvrir si vous avez vu juste. Voulez-vous reprendre la projection, s'il vous plaît?

(À suivre...)

Pour le lecteur critique et l'auteur de fiction que je suis, l'approche évolutionniste des œuvres - du passé et du présent - recèle d'innombrables inventions et redécouvertes. J'y vois non seulement une méthode permettant de mieux comprendre les comportements humains décrits par les œuvres narratives, mais aussi la perspective neuve d'écrire des fictions en s'amusant et de parler d'amour en toute consilience.

\section{Bibliographie}

Blaffer Hrdy, Sarah. (1999), Mother Nature. Natural Selection and the Female of the Species, Londres, Chatto \& Windus.

Boyd, Brian. (2009), On the Origin of Stories. Evolution, Cognition and Fiction, Cambridge, Belknap Press of Harvard University Press. 
-, Joseph CARroll et Jonathan GotTschall (eds.). (2010), Evolution, Literature \& Film. A reader, New York, Columbia University Press.

Buss, David M. (1995), The Evolution Of Desire. Strategies Of Human Mating, Jackson, Basic Books.

DutTon, Denis. (2009), The Art Instinct. Beauty, Pleasure and Human Evolution, New York, Bloombury Press.

GoTTSChaLL, Jonathan, David Sloan WiLSON (eds.). (2005), The Literary Animal. Evolution and the Nature of Narrative, Evanston, Northwestern University Press.

FISHER, Helen (1992), Anatomy of Love, New York, Fawcett Columbine.

Miller, Geoffrey. (2001), The Mating Mind. How Sexual Choice Shaped the Evolution of Human Nature, Londres, Random House Vintage.

-. (2009), Spent. Sex, Evolution and Consumer Behavior, New York, Viking Penguin.

SCALISE SugiYama, Michelle. (2010), « New Science, Old Myth: An Evolutionary Critique of the Oedipal Paradigm», dans B. Boyd, J. Carroll et J. Gottschall (eds.), Evolution, Literature \& Film.

Trivers, Robert L. (1971), «The Evolution of Reciprocal Altruism », The Quarterly Review of Biology, vol. 46 , no 1 , p. 35-57

WILSON, Edmund O. (1998), Consilience. The Unity of Knowledge, New York, Random House Vintage. 


\title{
Résumé
}

Des lectures évolutionnistes de Casablanca et de Cyrano de Bergerac et la description d'un projet romanesque actuellement en travail illustrent le changement de paradigme chez un auteur et critique de fiction francophone récemment émigré au Canada.

\begin{abstract}
A French-speaking fiction and non-fiction writer, recently settled in Canada, explains his paradigm shift to an evolutionary approach of literature through readings of Michael Curtiz's Casablanca and Edmond Rostand's Cyrano de Bergerac, and the description of a novel-in-progress.
\end{abstract}

